\title{
Microdosimetric Relative Biological Effectiveness of Therapeutic Proton Beams
}

\author{
Chuan-Jong Tung ${ }^{1,2}$
}

\begin{abstract}
When compared to photon beams, particle beams have distinct spatial distributions on the energy depositions in both the macroscopic and microscopic volumes. In a macroscopic volume, the absorbed dose distribution shows a rapid increase near the particle range, that is, Bragg peak, as particle penetrates deep inside the tissue. In a microscopic volume, individual particle deposits its energy along the particle track by producing localized ionizations through the formation of clusters. These highly localized clusters can induce complex types of deoxyribonucleic acid (DNA) damage which are more difficult to repair and lead to higher relative biological effectiveness (RBE) as compared to photons. To describe the biological actions, biophysical models on a microscopic level have been developed. In this review, microdosimetric approaches are discussed for the determination of RBE at different depths in a patient under particle therapy. These approaches apply the microdosimetric lineal energy spectra obtained

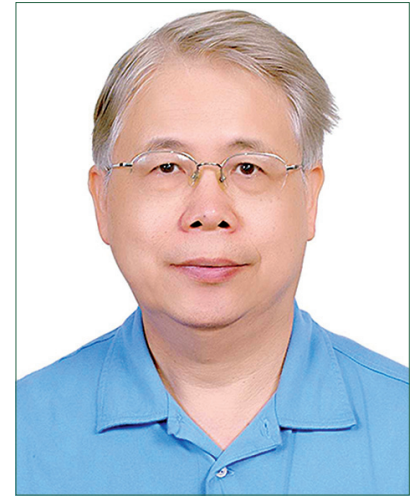

Prof. Chuan-Jong Tung from measurements or calculations. Methods to determine these spectra will be focused on the tissue equivalent proportional counter and the Monte Carlo program. Combining the lineal energy spectrum and the biological model, RBE can be determined. Three biological models are presented. A simplified model applies the dose-mean lineal energy and the measured RBE (linear energy transfer) data. A more detailed model makes use of the full lineal energy spectrum and the biological weighting function spectrum. A comprehensive model calculates the spectrum-averaged yields of DNA damages caused by all primary and secondary particles of a particle beam. Results of these models are presented for proton beams.
\end{abstract} (Biomed J 2015;38:399-407)

\section{Key words: microdosimetry, lineal energy, proton therapy, relative biological effectiveness}

$I_{n}^{n}$ n particle (proton or heavy-ion) therapy, the patient treatment planning includes assessments of radiation dose and radiation quality. Distributions of the absorbed dose and the relative biological effectiveness (RBE), a quality index, in the patient provide essential information in the treatment planning. To facilitate these assessments, the International Atomic Energy Agency and the International Commission on Radiation Units and Measurements (ICRU) have jointly recommended the reporting and recording of isoeffective doses in the treatment plan. ${ }^{[1]}$ The isoeffective dose, that is, the product of absorbed dose and isoeffective weighting factor, of particle beam is defined as the equivalent dose of photon beam under the same irradiation conditions (e.g., 2 Gy per fraction, 5 daily fractions per week, etc.). The key factor determining the isoeffective weighting factor is the RBE. While absorbed dose relates to the total energy deposition in a macroscopic volume of the irradiated tissue, radiation quality regards the single-event energy deposition in a microscopic volume of the biologically sensitive target. ${ }^{[2]}$

When compared to photon beams, particle beams have distinct spatial distributions on the energy depositions in both the macroscopic and microscopic volumes. ${ }^{[3]}$ Such distributions are responsible for the different biological consequences in the tumor and healthy cells during radiotherapy. The macroscopic distribution, that

From the ${ }^{1}$ Department of Medical Imaging and Radiological Sciences, College of Medicine, Chang Gung University, Taoyuan, Taiwan; ${ }^{2}$ Institute for Radiological Research, Chang Gung University/Chang Gung Memorial Hospital, Taoyuan, Taiwan

Received: Aug. 17, 2014; Accepted: Sep. 17, 2015

Correspondence to: Prof. Chuan-Jong Tung, Department of Medical Imaging and Radiological Sciences, College of Medicine, Chang Gung University, Taoyuan, Taiwan. 259, Wenhua $1^{\text {st }}$ Rd., Gueishan, Taoyuan 333, Taiwan (ROC). Tel.: 886-3-2118800 ext. 3614; Fax: 886-3-2118620; E-mail: cjtung@ mail.cgu.edu.tw

DOI: $10.4103 / 2319-4170.167072$ 
is, the depth-dose profile, of particle beams is largely influenced by the velocity-dependent stopping power, that is, the energy loss is inversely proportional to the particle velocity. As particle penetrates deep inside the tissue, particle velocity is decreasing so that stopping power rises, initially quite slow, and then very rapid near the particle range, to a maximum value at the Bragg peak. The Bragg peak feature makes particle beam particularly suitable for the treatment of deep-seated tumors. Besides the favorable dose distribution, individual particle deposits its energy along the particle track by producing localized ionizations in a microscopic volume through the formation of clusters. Experimental results demonstrated that deoxyribonucleic acid (DNA) double strand break (DSB) plays a key role in biological damages of the irradiated cell. ${ }^{[4]}$ Therefore, those particles with inelastic (ionization and excitation) mean free paths close to a few nanometers (the width of opposite DNA strands) are considered the most critical for the induction of lethal lesions. Such particles with linear energy transfer (LET) close to $100 \mathrm{keV} / \mu \mathrm{m}$ are most effective in inducing the biological effects.

Microdosimetry is a method that studies the pattern and magnitude of the microscopic energy deposition in a biological sensitive volume. ${ }^{[5]}$ Microdosimetry can be applied to predict the RBE of a given radiation type and energy by comparing these pattern and magnitude with those of the reference radiation, that is, ${ }^{60} \mathrm{Co}$ gamma rays for radiotherapy. In this review, microdosimetric approaches will be discussed for the determination of RBE values at different depths in a patient under particle therapy. These approaches apply the microdosimetric lineal energy spectra obtained from measurements or calculations. Methods to determine the microdosimetric spectra will be focused on the tissue equivalent proportional counter (TEPC), or miniature TEPC (mini-TEPC), and the Monte Carlo (MC) program. Combining a lineal energy spectrum and a biological model, RBE value can be determined. A simplified approach applies the dose-mean lineal energy and the measured RBE (LET) data. A more detailed approach makes use of the full lineal energy spectrum and biological weighting function spectrum. A comprehensive approach calculates the spectrum-averaged yields of DNA damages caused by all primary and secondary particles. Accordingly, RBE values at different depths in the patient can be estimated for any type of DNA damages, e.g., the DSB.

For proton beams with energies $70-250 \mathrm{MeV}$ used in proton therapy, the initial instantaneous LET values are less than $1 \mathrm{keV} / \mu \mathrm{m} .{ }^{[6]}$ Applying the measured RBE (LET) data, RBE values for proton beams at the entrance and plateau depths are all close to 1.0. At the distal edge of the Bragg peak, proton energies are slowed down to very low energies, yielding a maximum RBE value of 3.0-6.0, depending on the biological endpoint, corresponding to LET $\sim 90 \mathrm{keV} / \mu \mathrm{m}$. For clinical applications, this maximum RBE value exists only in a very narrow region where negligible biological significance is considered. Therefore, it is generally accepted that a constant RBE value of 1.1 is applied in proton therapy. For a precision dosimetry, variable RBE values near the distal edge of the Bragg peak can be considered. This could be the potential benefit of using proton therapy in Taiwanese cancer patients for the future. ${ }^{[7]}$

\section{MATERIALS AND METHODS}

Figure 1 is a schematic diagram showing the problems of the investigation. A proton beam transports in the tissue to a depth where the irradiation target is located. For proton therapy, two parameters are of special interest, that is, the absorbed dose, $D$, and the lineal energy, $y$. Absorbed dose is a measure of the total energy deposited in a macroscopic volume by the proton beam and all its secondary particles. Lineal energy is a measure of the single-event energy deposited in a microscopic volume of the biological target (e.g., cell nucleus or DNA). Comparing lineal energy spectra of the proton beam with those of the photon beam, RBE values at different depths in the tissue may be estimated. The lineal energy spectra may be obtained from either measurement using the TEPC or simulations using the MC code.

The lineal energy, $y$, is an analog of the LET. A comparison of LET and $y$ is depicted in Figure 2, where a proton loses its energy through electronic interactions (solid circles)

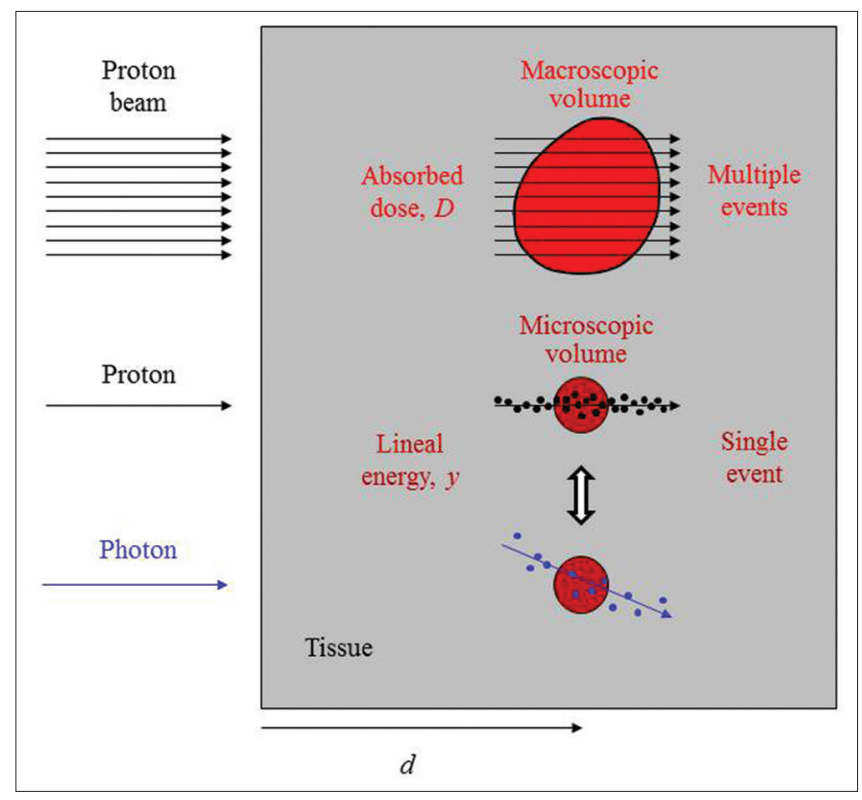

Figure 1: A schematic diagram showing the proton beam transporting in tissue. Absorbed dose, $D$ is a measure of the total energy deposition in a macroscopic volume. Lineal energy, $y$ is a measure of the single-event energy deposition in a microscopic volume of the biological target. Comparing lineal energy spectra of the proton beam with those of the photon beam, relative biological effectiveness values may be estimated. 


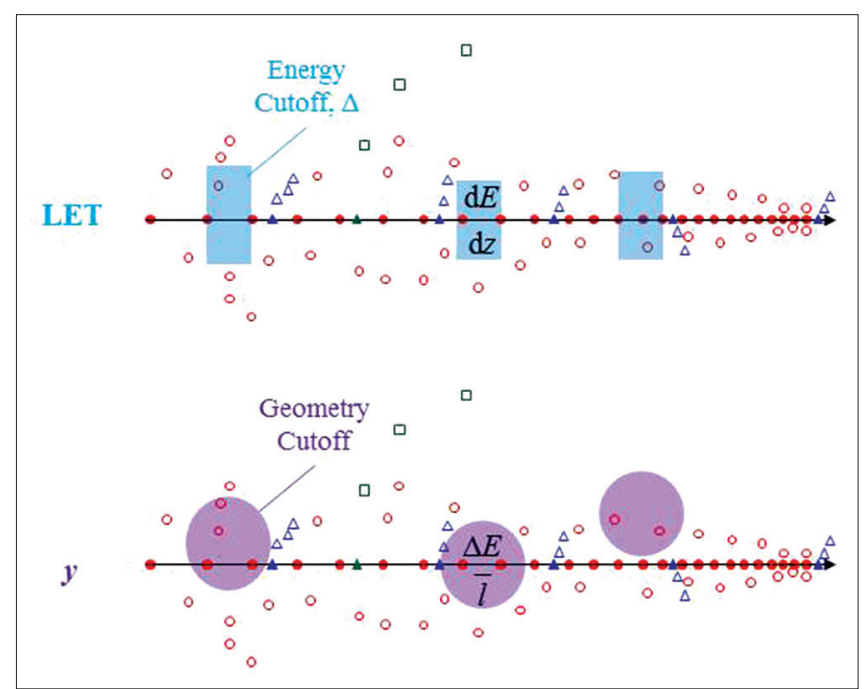

Figure 2: A proton loses its energy through electronic interactions (solid circles) by emitting secondary electrons (open circles) and through nuclear interactions (solid triangles) by generating charged particles (open triangles) and bremsstrahlungs (open squares). Linear energy transfer, $\mathrm{d} E / \mathrm{d} z$ is the mean energy loss per unit path length, subject to an energy-loss cut-off value $\Delta$. Lineal energy, $y$ is the energy deposition in a volume divided by the mean chord length of that volume, that is, $\Delta E / \overline{\mathrm{T}}$.

by emitting secondary electrons (open circles) and through nuclear interactions (solid triangles) by generating charged particles (open triangles) and bremsstrahlungs (open squares). The stopping power, $\mathrm{d} E / \mathrm{d} z$, defined as the mean total (electronic, nuclear, and radiative) energy loss per unit path length, is given by $S=S_{\text {ele }}+S_{\text {nuc }}+S_{\text {rad }}$. While the stopping power focuses on the total energy loss, LET restricts to the local (immediate proximity along the proton track) energy loss. ${ }^{[8]}$ Thus, delta-rays (high-energy secondary electrons), with energies larger than a cut-off value $\Delta$, which move away from the proton track are excluded in the definition of LET, or LET . Although $\mathrm{LET}_{\Delta}$ can be calculated, it cannot be measured because a detector corresponds to the geometry cut-off but not the energy cut-off. Therefore, it is more convenient to define $y$ as the ratio of energy deposited ( $\Delta E$ in the figure) in a volume (the detector or biological target, shaded sphere) and the mean chord length ( $\bar{\ell}$ in the figure) of that volume. ${ }^{[9]}$ The lineal energy can either be measured using a microdosimeter (e.g. a TEPC) or calculated using a $\mathrm{MC}$ simulation program.

For particle therapy, the isoeffective dose, $D_{\mathrm{IsoE}}$, is defined as the product of absorbed dose, $D$, and isoeffective-dose weighting factor, $W_{\text {IsoE }}$, that is, ${ }^{[1]}$

$$
D_{\text {IsoE }}=D \times W_{\text {IsoE }}
$$

Multiple variables affect the isoeffective-dose weighting factor, including the absorbed dose, dose rate, dose per fraction, radiation quality, and biological endpoint. Under the same irradiation conditions as used in photon therapy (e.g., 2 Gy per fraction, 5 daily fractions per week, etc.), radiation quality seems to be the key factor that determines the isoeffective-dose weighting factor of particle beam. Radiation quality refers to a characterization of the radiation type and energy for the induction of biological effects. The best description of radiation quality is the RBE. For a given biological endpoint, $\mathrm{RBE}$ is defined by ${ }^{[3]}$

$$
\operatorname{RBE}=\left.\frac{D_{\mathrm{r}}}{D_{\mathrm{p}}}\right|_{\text {Isoeffect }}
$$

where, $D_{\mathrm{p}}$ and $D_{\mathrm{r}}$ are absorbed doses of the particle radiation and the reference radiation (usually ${ }^{60} \mathrm{Co} \gamma$-rays), respectively. For proton therapy, ICRU recommends the use of a RBE-weighted absorbed dose: ${ }^{[10]}$

$$
D_{\mathrm{RBE}}=D \times \mathrm{RBE}
$$

As discussed in ICRU ${ }^{[10]}$ LET is a useful quantity in characterizing the biological effectiveness of an individual biological system. However, LET does not predict the biological response with high accuracy for different biological systems. Actually, LET is an approximation of the microdosimetric dose-mean lineal energy, $\bar{y}_{D}$ defined by

$$
\bar{y}_{D}=\int y d(y) \mathrm{d} y
$$

where, $d(y)$ is the normalized dose probability density as a function of lineal energy. Based on a simplified model, one can determine RBE from the database containing in vitro and in vivo results for a variety of biological effects. ${ }^{[10,11]}$ Assuming LET $=\bar{y}_{D}$, one determines the RBE using measured RBE (LET) data. Due to the variation of measured RBE (LET) data, it was previously suggested the upper and lower limits of measured data. ${ }^{[12]}$ Figure 3 shows the region of these data (shaded area), that is, bounded by the upper and lower limits. It reveals that RBE equals to 1.0 for $y<1$ and increases with $y$ for $y>1$. Applications of the lineal energy spectra to determine RBE can readily be achieved through the microdosimetry parameter $\bar{y}_{D}$. Figure 4 plots measured data of $\bar{y}_{D}$ using the TEPC ${ }^{[13]}$ and the mini-TEPC (open squares) and calculated results using the MC simulations (open circles). It indicates that $\bar{y}_{D}$ increases with decreasing proton energy, showing a steeper slope for proton energies below $40 \mathrm{MeV}$.

It is more comprehensive to apply the full lineal energy spectra to determine the RBE. In Figure 3, two biological weighting functions (right ordinate) are also plotted, one for the intestinal crypt regeneration, $r_{\mathrm{ICR}}(y)$ (solid curve), and the other for the DNA DSB, $r_{\mathrm{DSB}}(y)$ (dashed curve). Here, $r_{\text {ICR }}(y)$ is taken from experimental data of therapeutic neutron beams with different radiation qualities. ${ }^{[14]} r_{\mathrm{DSB}}(y)$ is derived using the MC Damage Simulation (MCDS) code for proton beams with different energies ${ }^{[15]}$ It is seen that both weighting functions fall in the region between upper 


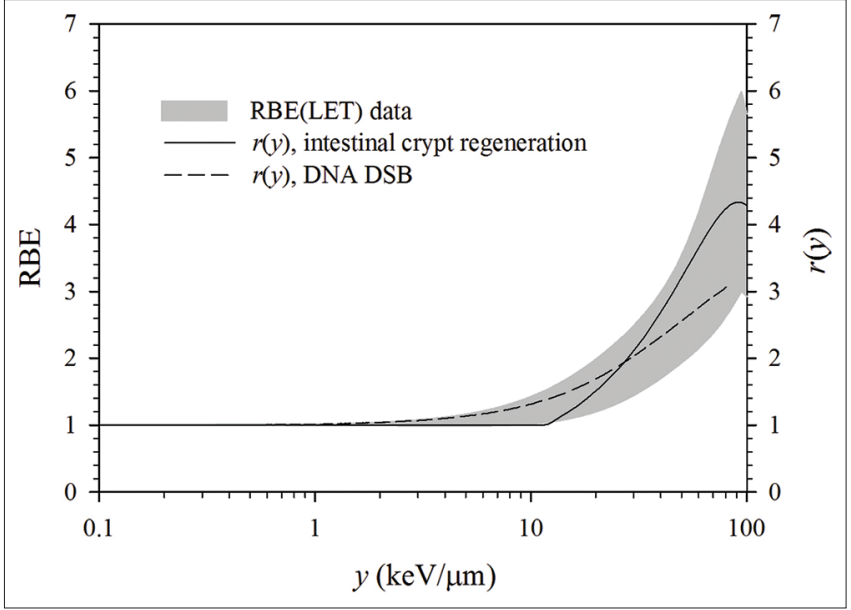

Figure 3: Relative biological effectiveness (linear energy transfer) data containing in vitro and in vivo results for a variety of biological effects. Due to the variation of these data, upper and lower limits are plotted (shaded area). Two biological weighting functions (right ordinate) are also plotted, one for the intestinal crypt regeneration (solid curve) and the other for the deoxyribonucleic acid double strand break (dashed curve).

and lower limits of RBE (LET) data. Below $\sim 25 \mathrm{keV} / \mu \mathrm{m}$, $r_{\text {ICR }}(\mathrm{y})<r_{\text {DSB }}(\mathrm{y})$; above $25 \mathrm{keV} / \mu \mathrm{m}, r_{\text {ICR }}(\mathrm{y})>r_{\text {DSB }}(\mathrm{y})$. Based on the biological weighting function, one can determine the $\mathrm{RBE}$ according to

$$
\mathrm{RBE}=\int d(y) \cdot r(y) \cdot \mathrm{d} y
$$

Equation (4) calculates the RBE from weighting the $y$-dependent biological function, $r(y)$, by the single-event dose spectrum, $d(y)$. Its validity was discussed in the applications to photons, protons, and neutrons. ${ }^{[16]}$ This equation was used to calculate the RBE for boron neutron capture therapy ${ }^{[17]}$ proton therapy, ${ }^{[18]}$ and heavy-ion therapy. ${ }^{[19]}$

Since lineal energy spectra are generally expressed in $y d(y)$ versus $\log (y)$, Equation (4) can be rewritten as:

$$
\mathrm{RBE}=\int y \cdot d(y) \cdot r(y) \cdot \mathrm{d}[\log (y)]
$$

For a given biological target, $d(y)$ can either be measured using a TEPC (or mini-TEPC) or simulated using an MC program. For instance, Figure 5 shows the mini-TEPC that can be used to measure the lineal energy spectra of proton beams. Panel (A) is the complete mini-TEPC, with the detector (at the left end) enclosed in an aluminum housing that serves as a vacuum tight container. Panel (B) is a perspective view of the mini-TEPC with all components and dimensions. The $1 \mathrm{~mm} \times 1 \mathrm{~mm}$ (height $\times$ diameter) cylindrical cavity in the A- 150 plastic is the sensitive volume that actually detects the ionization signal. The anode is a gold-plated tungsten wire stretched by a spring. In addition to experimental measurements, MC transport codes can be employed to determine the

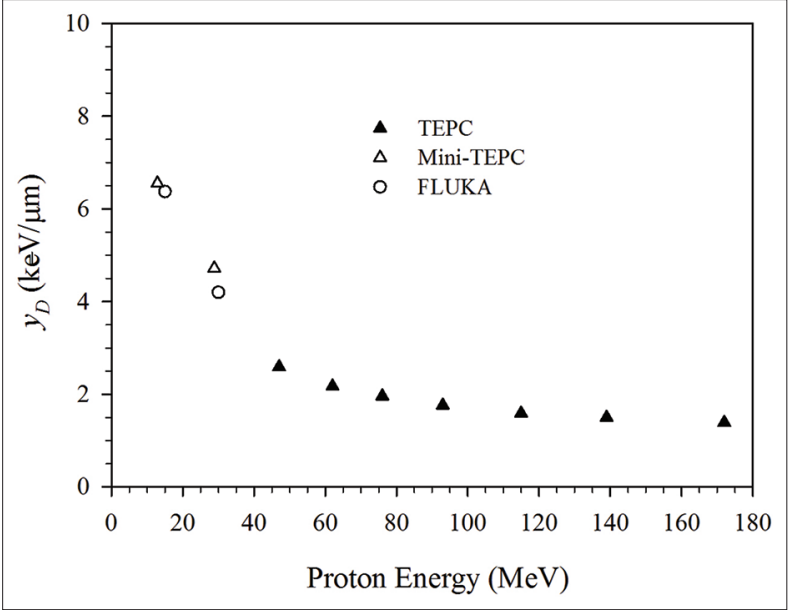

Figure 4: Dose-mean lineal energies for protons measured by the tissue equivalent proportional counter (solid triangles) and the mini-tissue equivalent proportional counter (open triangles) and calculated by the Monte Carlo simulations (open circles).

lineal energy spectra. Studies demonstrated that MC FLUKA $\operatorname{code}^{[20,21]}$ was able to predict the spectra of proton beams ${ }^{[22]}$ and heavy-ion beams. ${ }^{[23]}$ In the simulations, energy losses, energy loss straggling, scattering, and nuclear interactions of all primary and secondary particles can be included. To increase the efficiency of simulations, the microscopic tissue target can be expanded in volume by the application of Fano theorem, ${ }^{[24]}$ that is, the flux of secondary radiation is independent of the density of a medium with given compositions.

In Figure 3, the biological weighting function for DNA DSB is calculated for protons using the MCDS program. For a proton beam moving in the patient, however, secondary nuclear particles are generated. Therefore, all primary and secondary particles must be included in the determination of proton beam RBE. Because different particles contribute different biological effectiveness, an overall RBE of the proton beam is then calculated by a fluence-averaged RBE. The MCDS code provides a simple algorithm that simulates the DNA clustered damages induced by ionizing radiations of different qualities. This algorithm calculates the yields of different types of DNA damages. The DNA damage yield for a mixture of primary and secondary particles can then be estimated by weighting the corresponding yield by the fluence spectrum of each type of particle. The fluence spectra of all primary and secondary particles at a given depth can be calculated using the FLUKA code. Let the

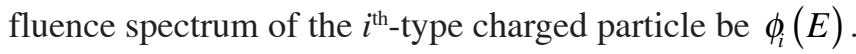
Combine this spectrum with the DNA damage yield of the $j^{\text {th }}$-type (e.g., DSB) per cell, $Y_{i j}(E)$ through the relation ${ }^{[25]}$

$$
Y_{i j}=\frac{\int \mathrm{d} E \cdot Y_{i j}(E) \cdot \phi_{i}(E) \cdot \operatorname{LET}_{i}(E)}{\int \mathrm{d} E \cdot \phi_{i}(E) \cdot \operatorname{LET}_{i}(E)}
$$




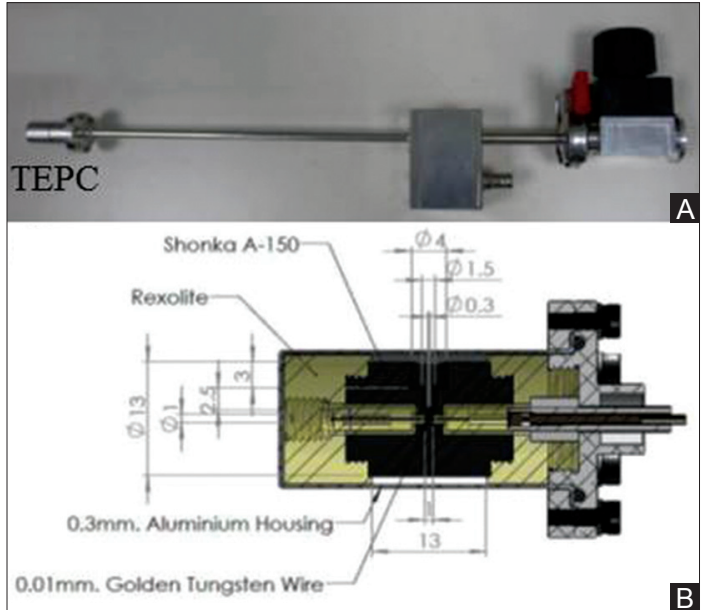

Figure 5: Photographs of the mini-tissue equivalent proportional counter. Panel (A) complete mini-tissue equivalent proportional counter, with the detector (far left end) enclosed in an aluminum housing. Panel (B) perspective view of the mini-tissue equivalent proportional counter with components and dimensions.

Where, $\operatorname{LET}_{i}(E)$ is the LET as a function of energy, $E$ for the $i^{\text {th }}$-type particle. The RBE for the $j^{\text {th }}$-type DNA damage relative to ${ }^{60} \mathrm{Co}$ for the $i^{\text {th }}$-type particle is determined from

$$
\mathrm{RBE}_{i j}=\frac{Y_{i j}}{Y_{j}\left({ }^{60} \mathrm{Co}\right)}
$$

where, $Y_{j}\left({ }^{60} \mathrm{Co}\right)$ is the corresponding average yield of $j^{\text {th }}$-type DNA damage for a uniformly distributed ${ }^{60} \mathrm{Co}$ source. The RBE for the $j^{\text {th }}$-type DNA damage relative to ${ }^{60} \mathrm{Co}$ for the proton beam is then calculated using

$$
\mathrm{RBE}_{j}=\frac{\sum_{\mathrm{i}}\left(\mathrm{RBE}_{i j} \times D_{i}\right)}{\sum_{i} D_{i}}
$$

Where, $D i$ is the absorbed dose from the $i^{\text {th }}$-type particle and the summation is over all primary and secondary particles of the proton beam.

\section{RESULTS AND DISCUSSION}

Figure 6 shows the results of dose-weighted lineal energy spectra, $y d(y)$, measured using the mini-TEPC and simulated using the FLUKA code for, nominally, 15 and $30 \mathrm{MeV}$ proton beams emitted from the medical cyclotron TR 30/15. ${ }^{[26]}$ It is seen that the shapes of measured spectra are generally reproduced by the MC simulations. Notice that in mini-TEPC measurements proton energies at the detector are less than those emitted from the cyclotron, because of the slowing-down and straggling of protons in the mini-TEPC Al case. Thus, lineal energy spectra measured by the mini-TEPC represent results for proton energies of 12.88 and $28.83 \mathrm{MeV}$, corresponding to 15 and $30 \mathrm{MeV}$ emitted energies. This makes the main peaks of measured

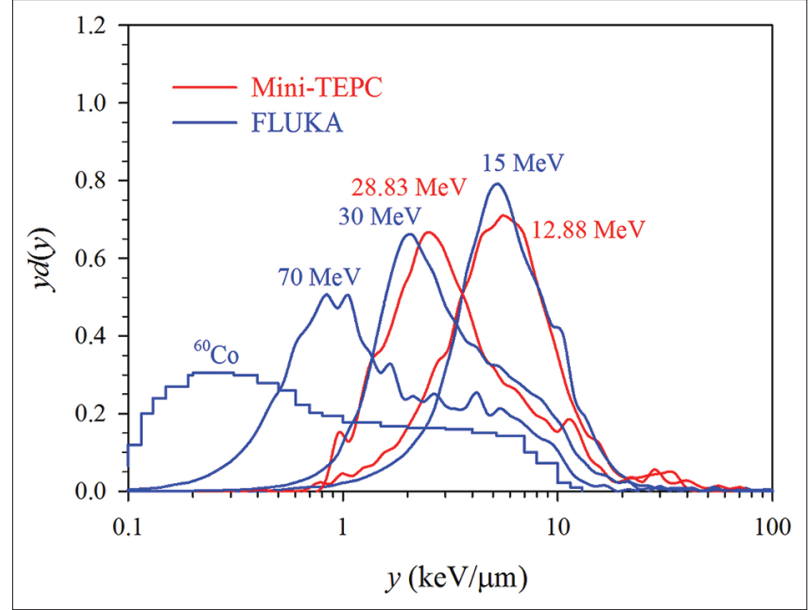

Figure 6: Dose-weighted lineal energy spectra, $y d(y)$ obtained from the mini-tissue equivalent proportional counter measurements and the Monte Carlo simulations for, nominally, 15 and $30 \mathrm{MeV}$ proton beams. Monte Carlo simulated results of $70 \mathrm{MeV}$ protons and ${ }^{60} \mathrm{Co} \gamma$-rays are also shown.

data shift to higher lineal energies compared to simulated results. The spectra at $y>10 \mathrm{keV} / \mu \mathrm{m}$ correspond to scattered protons in the mini-TEPC wall, revealing a maximum attainable lineal energy $\sim 140 \mathrm{keV} / \mu \mathrm{m}$, that is, the proton edge. ${ }^{[27]}$ In this figure, MC simulated spectra of $70 \mathrm{MeV}$ protons are also plotted. It is seen that lineal energy spectra move to higher $y$-values as proton energy decreases. It is also seen that proton lineal energy spectra are mainly distributed between 1 and $10 \mathrm{keV} / \mu \mathrm{m}$, as compared to the ${ }^{60} \mathrm{Co} \gamma$-ray spectrum between 0.1 and $1 \mathrm{keV} / \mu \mathrm{m} .{ }^{[22]}$ As proton energy decreases, a greater portion of the spectra falls in the range of lineal energy larger than $10 \mathrm{keV} / \mu \mathrm{m}$.

MC simulated results of the absorbed dose distribution in a water phantom for $70 \mathrm{MeV}$ proton beam are plotted in Figure 7 (left panel). Here the Bragg peak at $40 \mathrm{~mm}$ depth is clearly seen. This peak is beneficial in the treatment of ocular melanoma. Lineal energy spectra of $1 \mu \mathrm{m}$-diameter target, $d(y)$, at various depths in the phantom (labeled as circles) are also plotted in Figure 7 (right panel). As depth is increasing, a progressive shift of the lineal energy spectrum toward higher $y$-value is seen. This shift is responsible for the RBE increase, with depth, at the distal edge of the Bragg peak, when compared with RBE values at the plateau and shallower depths. Applying the lineal energy spectra to the RBE (LET) data or the biological weighting functions, RBE values at various depths in the water phantom can be estimated. RBE values determined from using the upper and lower limits of RBE (LET) data are plotted in Figure 8, along with the depth-dose curve for $70 \mathrm{MeV}$ proton beam. Corresponding RBE values obtained using the biological weighting function for the intestinal crypt regeneration are also plotted in this figure. It is seen that RBE values increase rapidly at the distal edge of the Bragg peak, reaching a 


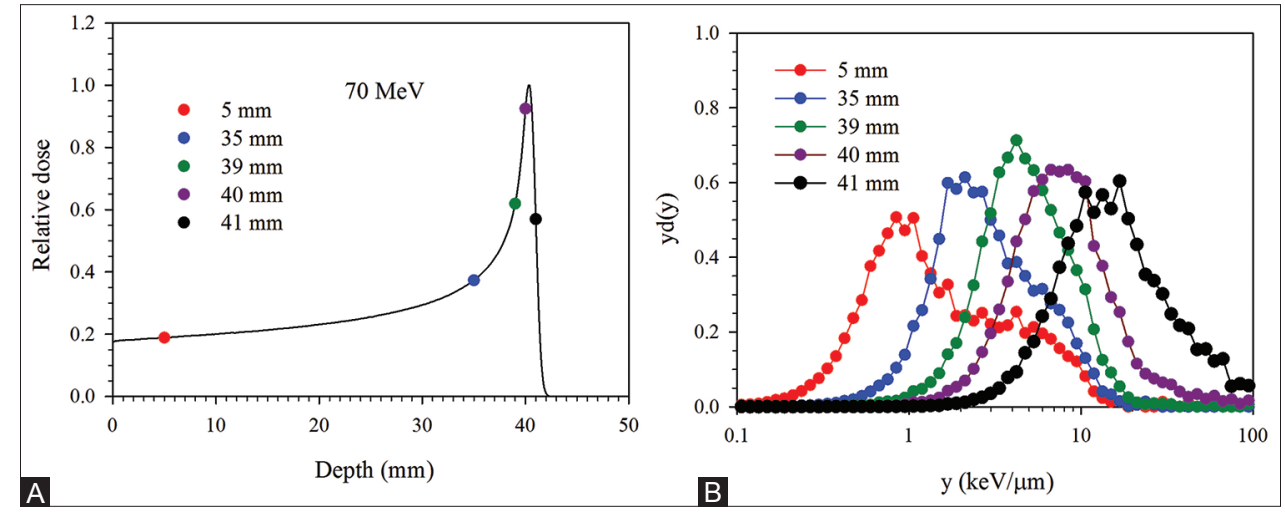

Figure 7: (A) Monte Carlo simulated results of the absorbed dose distribution in a water phantom for $70 \mathrm{MeV}$ proton beam. (B) Lineal energy spectra of $1 \mu \mathrm{m}$-diameter target, $d(y)$ at various depths, labelled in (A), in the phantom.

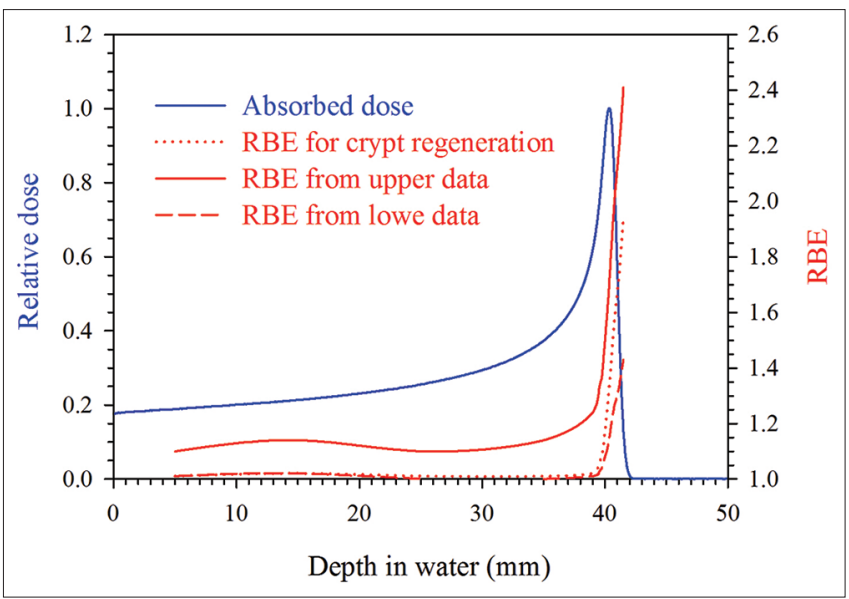

Figure 8: Relative biological effectiveness values determined from $\bar{y}_{D}$ using the upper and lower limits of relative biological effectiveness (linear energy transfer) data, and the depth-dose curve for $70 \mathrm{MeV}$ proton beam. Relative biological effectiveness values obtained using the biological weighting function for the intestinal crypt regeneration is also shown.

maximum value $>2.0$. The biological weighting function method yields RBE values in the range bounded by the upper and lower limits.

For DNA DSB, the MCDS program simulates the fluence-averaged RBE according to Equations (4-6). Figure 9 shows the depth-dose distributions calculated using the FLUKA code for $160 \mathrm{MeV}$ proton beam in the water phantom. Individual contributions to the total dose from primary protons and secondary particles (secondary protons, alpha-particles, deuterons, and He3-ions) are separately plotted. It is seen that the contributions from secondary particles are almost invisible near the Bragg peak, but are marked in the plateau region and at depths beyond the distal edge of the peak. The contributions of secondary protons, alpha-particles, deuterons, and He3-ions differ by orders of magnitude. The contribution from primary protons terminates at a maximum depth close to the distal edge of the Bragg peak. Secondary particles, however, continue to make contributions beyond the distal edge of the peak.

Using FLUKA, the fluence spectra of primary and secondary particles at several depths in the water phantom are plotted in Figure 10 for $160 \mathrm{MeV}$ proton beam. The primary proton spectrum shows a narrow peak around $160 \mathrm{MeV}$ at $0.5 \mathrm{~cm}$ and a broad peak around $15 \mathrm{MeV}$ at $17.475 \mathrm{~cm}$ (the Bragg peak depth). The broader and lower spectra of primary protons at deeper depths are due to the energy loss and straggling. Because of the greatly reduced proton energies, LET and RBE at the Bragg peak are expected to go up sharply. On the other hand, the spectra of secondary particles continue to fall with increasing depth due to the smaller number of primary protons and so less nuclear interactions. Although secondary particles have smaller energies or higher LET than primary protons, their numbers are less. Therefore, the contribution from secondary particles to the RBE is expected to be small. Using MCDS, RBE values for the induction of DSB are plotted in Figure 11 for $160 \mathrm{MeV}$ proton beam (right ordinate). It is seen that RBE increases slowly from the entrance depth and rapidly near the Bragg peak. The maximum RBE value at $0.5 \mathrm{~cm}$ distal to the Bragg peak is equal to 1.5. RBE-weighted absorbed doses (left ordinate) were plotted and compared with absorbed doses. The effect that RBE increases with decreasing dose beyond the Bragg peak is the extension of dose range by $\sim 1.9 \mathrm{~mm}$.

A spread out Bragg peak (SOBP) between 14.0 and $17.3 \mathrm{~cm}$ is made up of proton beams of successively lower energies. RBE values for the induction of DSB for the SOBP proton beam are also calculated using the MCDS code. Calculated RBE values (right ordinate), absorbed doses (left ordinate), and RBE-weighted absorbed doses (left ordinate) are plotted in Figure 12. Again, close to the distal edge of the SOBP, the enhancement of RBE-weighted absorbed doses is more pronounced. In this figure, RBE values for the $160 \mathrm{MeV}$ unmodulated proton beam are also included for comparisons. It reveals that RBE values are greater for modulated beam than unmodulated beam at depths proximal 


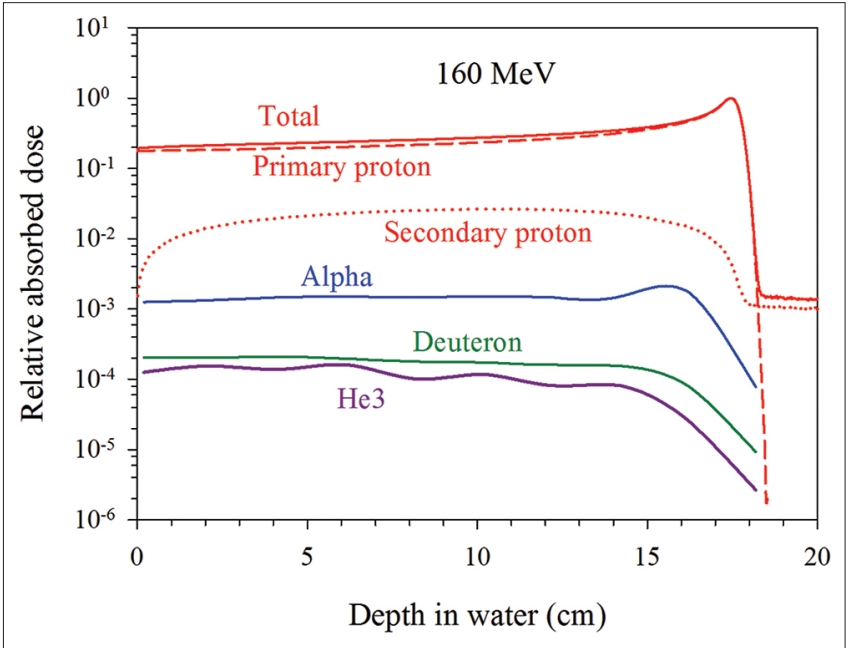

Figure 9: The depth-dose distribution for a $160 \mathrm{MeV}$ proton beam normally incident on a water phantom. Individual contributions from primary protons and secondary particles are also plotted. All doses are normalized to the total dose at the Bragg peak.

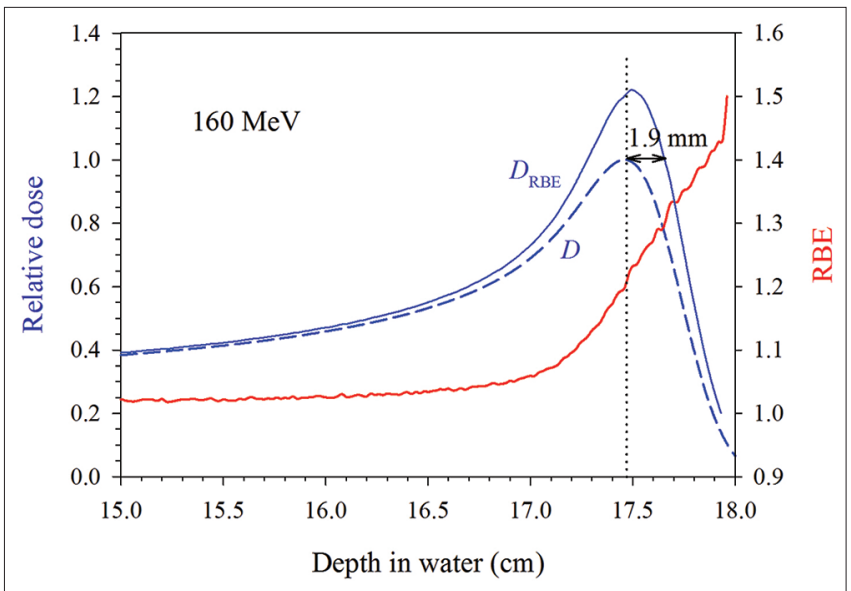

Figure 11: A comparison of absorbed dose, $D$ (dashed curve, left ordinate) and relative biological effectiveness-weighted absorbed dose, $D_{\text {RBE }}$ (solid curve, left ordinate) for a $160 \mathrm{MeV}$ proton beam normally incident on a water phantom. All doses are normalized to the absorbed dose at the Bragg peak (dotted line). Relative biological effectiveness values for the induction of deoxyribonucleic acid double strand break are also plotted (right ordinate).

to the Bragg peak. On the contrary, RBE values are greater for unmodulated beam than modulated beam at depths distal to the Bragg peak. This is easily understood because RBE for a modulated proton beam is the average of all RBE values for many lower energy unmodulated proton beams.

\section{Conclusions}

When compared to photon beam, charged particle beam retains a better absorbed dose distribution and a larger biological effectiveness to maintain the merits of particle therapy. The highly localized energy deposition of particles

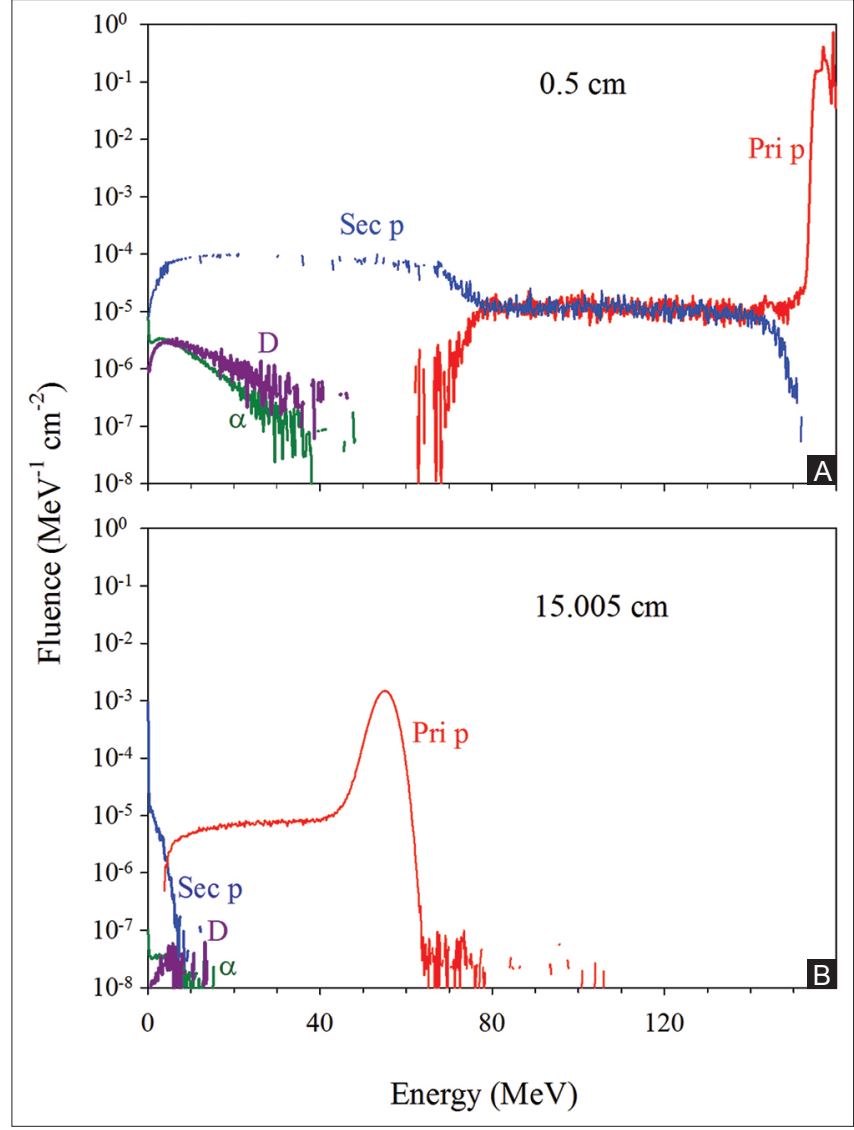

Figure 10: (A) The fluence spectra of primary protons and secondary particles at $0.5 \mathrm{~cm}$ depth in the water phantom for a $160 \mathrm{MeV}$ proton beam. (B) Similar spectra at $15.005 \mathrm{~cm}$ depth.

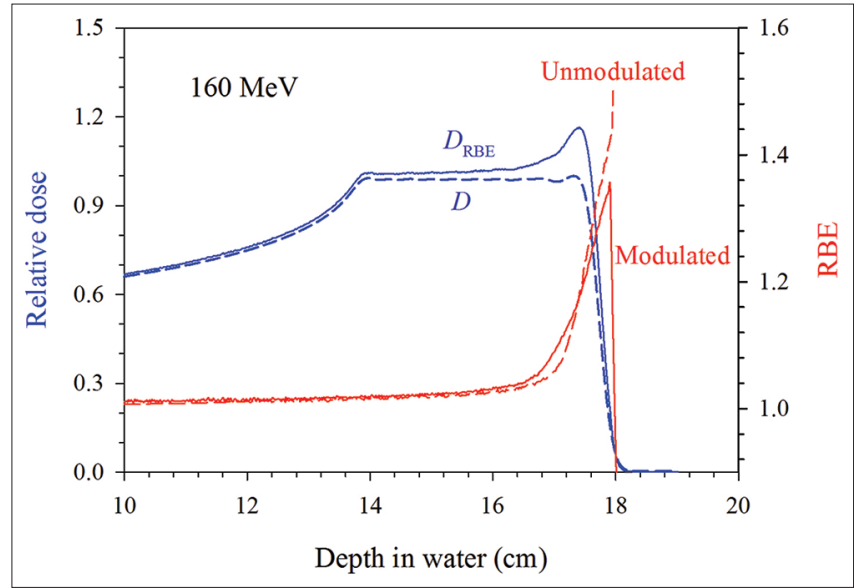

Figure 12: A comparison of absorbed dose, $D$ and relative biological effectiveness-weighted absorbed dose, $D_{\mathrm{RBE}}$ for a modulated $160 \mathrm{MeV}$ proton beam with SOBP between 14.0 and $17.3 \mathrm{~cm}$. Relative biological effectiveness values for the induction of deoxyribonucleic acid double strand break (right ordinate) of the corresponding modulated and unmodulated beams are also plotted for comparison.

induces complex types of DNA damage. Since these types of damage are more difficult to repair, particles should 
have higher RBE values, that is, requiring lower doses to achieve the same biological effects as compared to photons. To describe the biological actions, biophysical models on a microscopic level have been developed. Although these models involve crude approximations and mostly focus on the specific aspect of radiation action, they are very useful in the application of particle therapy. The empirical approaches including biological weighting function model and fluence-averaged DNA damage model provide satisfactory quantitative agreement with experimental data. The increased biological effectiveness of particle beam has to be taken into account in the treatment planning.

For proton beams with energies $70-250 \mathrm{MeV}$ used in proton therapy, maximum RBE values at the distal edge of the Bragg peak are theoretically estimated to be 3.0-6.0 based on the RBE (LET) data, depending on the biological endpoint. For clinical applications, these maximum RBE values exist only in a very narrow region where negligible biological significance is of concern. Therefore, it is generally accepted that a constant RBE value of 1.1 is applied in proton therapy. However, this does not exclude the need of a precision dosimetry, where variable RBE values in the patient need to be considered. In this review, microdosimetric approaches have been discussed for the determination of RBE values at different depths in a patient under particle therapy. These approaches apply the lineal energy spectra obtained from TEPC measurements or MC calculations. Combining a lineal energy spectrum and a biological model, RBE value can be determined. Results of these approaches have been presented for proton beams of 15/30 MeV, $70 \mathrm{MeV}$, and $160 \mathrm{MeV}$.

\section{Acknowledgment}

This research was supported by the Chang Gung Medical Research Program (CIRPD1C0013, CIRPD1C0033) and the National Science Council, Taiwan. (NSC 101-2314-B182-058).

\section{Conflicts of interest}

There are no conflicts of interest.

\section{REFERENCES}

1. International Atomic Energy Agency. IAEA-TECDOC-1560. Vienna: International Atomic Energy Agency; 2007.

2. International Commission on Radiation Units and Measurements. ICRU Report 85. Oxford: Oxford University Press; 2011.

3. Scholz M. Dose response of biological systems to low- and high-LET radiation. In: Horowitz Y, editor. Microdisemetric Response of Physical and Biological Systems to Low- and High-LET Radiations. Amsterdam: Elsevier; 2006. p. 1-73.

4. Frankenberg D, Frankenberg-Schwager M, Blöcher D, Harbich R. Evidence for DNA double-strand breaks as the critical lesions in yeast cells irradiated with sparsely or densely ionizing radiation under oxic or anoxic conditions. Radiat Res 1981;88:524-32.

5. Rossi HH, Zaider M. Microdosimetry and Its Applications. Berlin: Springer-Verlag; 1996.

6. Berger MJ, Coursey JS, Zucker MA, Chang J. Stopping-Power and Range Tables for Electrons, Protons, and Helium Ions. National Institute of Standards and Technology, Gaithersburg, Maryland, USA. Available from: http://www.physics.nist.gov/PhysRefData/Star/Text/ PSTAR.html. [Last updated on 2011 Dec 09].

7. Kao WH, Shen YL, Hong JH. What are the potential benefits of using proton therapy in Taiwanese cancer patients? Biomed J 2015;38:391-8.

8. International Commission on Radiation Units and Measurements. ICRU Report 16. Oxford: Oxford University Press; 1970.

9. International Commission on Radiation Units and Measurements. ICRU Report 36. Oxford: Oxford University Press; 1983.

10. International Commission on Radiation Units and Measurements. ICRU Report 78. Oxford: Oxford University Press; 2007.

11. Loeffler JS, Durante M. Charged particle therapy - Optimization, challenges and future directions. Nat Rev Clin Oncol 2013;10:411-24.

12. Chu WT, Ludewigt BA, Renner TR. Instrumentation for treatment of cancer using proton and light-ion beams. Rev Sci Instrum 1993;64:2055-122.

13. Borak TB, Doke T, Fuse T, Guetersloh S, Heilbronn L, Hara K, et al. Comparisons of LET distributions for protons with energies between 50 and $200 \mathrm{MeV}$ determined using a spherical tissue-equivalent proportional counter (TEPC) and a position-sensitive silicon spectrometer (RRMD-III). Radiat Res 2004;162:687-92.

14. Pihet P, Menzel HG, Schmidtz R, Beauduin M, Wambersie A. Biological weighting function for RBE specification of neutron therapy beams. Radiat Prot Dos 1990;31:437-42.

15. Semenenko VA, Stewart RD. Fast Monte Carlo simulation of DNA damage formed by electrons and light ions. Phys Med Biol 2006;51:1693-706.

16. Loncol T, Cosgrove V, Denis JM, Gueulette J, Mazal A, Menzel HG, et al. Radiobiological effectiveness of radiation beams with broad LET spectra: Microdosimetric analysis using biological weighting functions. Radiat Prot Dosimetry 1994;52:347-52.

17. Hsu FY, Tung CJ, Watt DE. Microdosimetric spectra of the THOR neutron beam for boron neutron capture therapy. Radiat Prot Dosimetry 2003;104:121-6.

18. Coutrakon G, Cortese J, Ghebremedhin A, Hubbard J, Johanning J, Koss $\mathrm{P}$, et al. Microdosimetry spectra of the Loma Linda proton beam and relative biological effectiveness comparisons. Med Phys 1997;24:1499-506.

19. Gerlach R, Roos H, Kellerer AM. Heavy ion RBE and microdosimetric spectra. Radiat Prot Dosimetry 2002;99:413-8.

20. Battistoni G, Muraro S, Sala PR, Cerutti F, Ferrari A, Roesler S, et al. The FLUKA code: Description and benchmarking. In: Albrow M, Raja R, editors. Proceedings of the Hadronic Shower Simulation Workshop; 6-8 September, 2006. Vol. 896. Fermilab: AIP Conference Proceeding; 2007. p. 31-49.

21. Ferrari A, Sala PR, Fasso A, Ranft J. FLUKA: A Multi-particle Transport code. CERN-2005-10, INFN/TC_05/11, SLAC-R-773; Stanford Linear Accelerator Center, Stanford University, Stanford, 
CA 94309, USA; 2005.

22. Rollet S, Colautti P, Grosswendt B, Moro D, Gargioni E, Conte V, et al. Monte Carlo simulation of mini TEPC microdosimetric spectra: Influence of low energy electrons. Radiat Meas 2010;45:1330-3.

23. Böhlen TT, Dosanjh M, Ferrari A, Gudowska I. Simulations of microdosimetric quantities with the Monte Carlo code FLUKA for carbon ions at therapeutic energies. Int $\mathrm{J}$ Radiat Biol 2012;88:176-82.

24. Fano U. Note on the Bragg-Gray cavity principle for measuring energy dissipation. Radiat Res 1954;1:237-40.

25. Wang CC, Hsiao Y, Lee CC, Chao TC, Wang CC, Tung CJ. Monte Carlo simulations of therapeutic proton beams for relative biological effectiveness of double-strand break. Int J Radiat Biol 2012;88:158-63.

26. Pan CY, Huang YW, Cheng KH, Chao TC, Tung CJ. Microdosimetry spectra and relative biological effectiveness of 15 and $30 \mathrm{MeV}$ proton beams. Appl Radiat Isot 2015;97:101-5.

27. International Commission on Radiation Units and Measurements. ICRU Report 49. Oxford: Oxford University Press; 1993.

This is an open access article distributed under the terms of the Creative Commons Attribution-NonCommercial-ShareAlike 3.0 License, which allows others to remix, tweak, and build upon the work non-commercially, as long as the author is credited and the new creations are licensed under the identical terms.

For reprints contact: reprints@medknow.com 